\title{
RECONSTRUÇÃO ÓSSEA EM MAXILA ATRÓFICA COM ENXERTO DE CRISTA ILÍACA: RELATO DE CASO
}

BONE RECONSTRUCTION IN ATROPHIC JAW WITH ILIAC CREST GRAFT: A CASE REPORT

Tâmara Pereira Brito ${ }^{1}$, Ramon dos Santos Nascimento ${ }^{1 *}$, Eduardo Francisco de Deus Borges², Christiano Sampaio Queiroz ${ }^{1}$, André Sampaio Souza ${ }^{1}$.

${ }^{1}$ Centro Universitário Ruy Barbosa, Salvador, BA, Brasil.

${ }^{2}$ Universidade Federal da Bahia, Salvador, BA, Brasil.

*ramonsantos110694@gmail.com

Recebido em: 17/12/2019; Aceito em: 07/10/2020.

\section{RESUMO}

O edentulismo total, apesar de toda evolução da Odontologia, ainda é uma condição comum na atualidade.Os tratamentos para esse tipo de patologia mais utilizados são: prótese muco suportada e implanto suportada. Em ambas as opções, a perda óssea pode inviabilizar o tratamento. São vários os tipos de biomateriais disponíveis para enxertia, podendo ser classificados como: autógenos, homólogos, heterógeno, e aloplástico. O objetivo do trabalho é relatar o caso clínico de um paciente com maxila atrófica severa, realizada a reconstrução óssea com enxerto autógeno removido da crista ilíaca em ambiente hospitalar e sob anestesia geral. Observou-se como principal vantagem a disponibilidade do material para enxertia, biocompatibilidade e rápida incorporação do enxerto.

Palavras-chave: Biomateriais. Crista ilíaca. Implantodontia. Reconstrução óssea.

\section{ABSTRACT}

Total edentulism, despite all the evolution of dentistry, still a common condition actualy. The most commonly used treatments for this type of pathology are: supported mucus prosthesis and supported implant. In both options, bone loss may make the treatment unfeasible. There are several types of biomaterials available for grafting, which can be classified as: autogenous, counterpart, heterogeneous, and alloplastic. The objective of this study is to report the clinical case of a patient with severe atrophic maxilla, who fulfilled the bone reconstruction with autogenous graft removed from the iliac crest in a hospital setting and under general anesthesia. It was observed the main advantage, the availability of the material for grafting, biocompatibility and rapid incorporation of the graft.

Keywords: Biomaterials. Bone reconstruction. Iliac crest. Implantology. 


\section{INTRODUÇÃO}

A implantodontia contemporânea foi idealizada por BRANEMARK como opção de tratamento para os pacientes com ausências dentárias que tenham dificuldades em utilizar uma prótese convencional, dando qualidade de vida, trazendo maior eficácia mastigatória, conforto e favorecimento no aspecto psicológico (PALECKIS et al., 2005).

A reabsorção óssea alveolar é um processo que pode ocorrer devido a perda dentária ao longo da vida tornando-se um fator limitante para a reabilitação com implantes dentários. A atrofia dos maxilares pode causar 0 comprometimento da função mastigatória, envelhecimento precoce, perda deretenção e alteração da estabilidade protética (WORTHINGTON; LANG; RUBENSTEIN, 2005).

Para a reabilitação de pacientes edêntulos com atrofia maxilar é necessário o tratamento prévio com enxerto ósseo, visando o aumento do rebordo alveolar (FLORIAN et al., 2010), que tem a principal função da reconstrução óssea e o favorecimento da posição dos implantes osseointegrados nos rebordos edêntulos, tendo a eficiência e durabilidade da reabilitação protética, recompondo o sistema estomatognático em função e estética (LEVIN; NITZAN; ARAD, 2007).

Os enxertos ósseos podem ser classificados como: autógeno, quando o material é removido do próprio indivíduo; homólogo,quando for doado por um indivíduo da mesma espécie; heterógeno, quando o material for retirado de um indivíduo de outra espécie; aloplástico, quando o material é produzido sinteticamente (HALLMAN; THOR, 2008).

Entre os tipos de enxertos existentes, o considerado padrão ouro dos cirurgiões dentistas é o autógeno, devido a biocompatibilidade e por ter capacidade osteogênese, osteoindução e osteocondução (PREJRONE et al., 2002). Porém, sua maior desvantagem é que precisa de outro sítio cirúrgico para obtenção do enxerto, ou seja, o paciente terá duas feridas.

As principais áreas doadoras para a enxertia autógena podem ser divididas em intrabucal: túber da maxila, mento e região retromolar e a extrabucal: calota craniana, fíbula e crista ilíaca, onde o local da escolha depende da quantidade de osso necessário para a reconstrução. Em local de pequeno e médio defeito escolhe o intrabucal, para reconstrução de áreas extremas, a área doadora extrabucal torna se mais indicada (SILVA et al., 2007; SOUZA et al., 2019).

Por fornecer uma grande quantidade de enxerto ósseo medular e córticomedular, sendo suficiente para reconstruções maxilares grandes em espessura, altura e para elevação bilateral do assoalho do seio maxilar, a crista ilíaca é utilizada como uma das opções do enxerto extrabucal que tem como vantagens, maior quantidade de material, incorporação do enxerto de forma rápida e previsível. Entretanto, os índices de complicações trans e pós-operatórios são baixos e geralmente relacionados com a quantidade de osso removido, podendo ocorrer hemorragia interna, edemas, dor, ruptura do nervo lateral femoral cutâneo, que provoca parestesia parcial ou definitiva na porção lateral da coxa e dificuldade de locomoção (SJÖSTRÖM et al., 2007; SBORDONE et al., 2009). 
O paciente deve se submeter a uma intervenção hospitalar com anestesia geral e com uma equipe composta por um bucomaxilofacial juntamente com uma equipe de ortopedia, que se encarregará da remoção do enxerto da crista ilíaca (SILVA et al., 2007).

O objetivo do trabalho é relatar o caso clínico de um paciente com maxila atrófica severa, sendo realizada a reconstrução óssea com enxerto autógeno removido da crista ilíaca, em ambiente hospitalar e sob anestesia geral.

\section{RELATO DE CASO}

Paciente M.G.S, sexo masculino, 48 anos, casado, atua na profissão de técnico de radiologia, compareceu a clínica do CEBEO Bahia na pós graduação de implantodontia, informando ter feito um tratamento prévio de um implante, porém não obteve sucesso devido a perda óssea. O mesmo nega problemas sistêmicos, tabagismo e etilismo e informa não ter nenhuma alergia a medicamentos

O paciente já havia sido submetido a procedimentos de enxertia óssea e de implantes, porém sem sucesso, e referia perda de 4 implantes na fase de instalação de cicatrizadores. Após avaliação clínica e radiográfica, foi possível avaliar a dimensão do defeito ósseo, o que levou à decisão pela reconstrução maxilar com enxerto de crista óssea.

$\mathrm{Na}$ Figura 1a, temos uma imagem clínica onde foi observado um grande defeito ósseo com as bordas do rebordo irregulares, ou seja, uma atrofia maxilar severa. Na Figura 1b, temos a imagem da avaliação radiográfica com defeito ósseo.

Figura 1 - Avaliação clínica (a) e radiográfica (b)
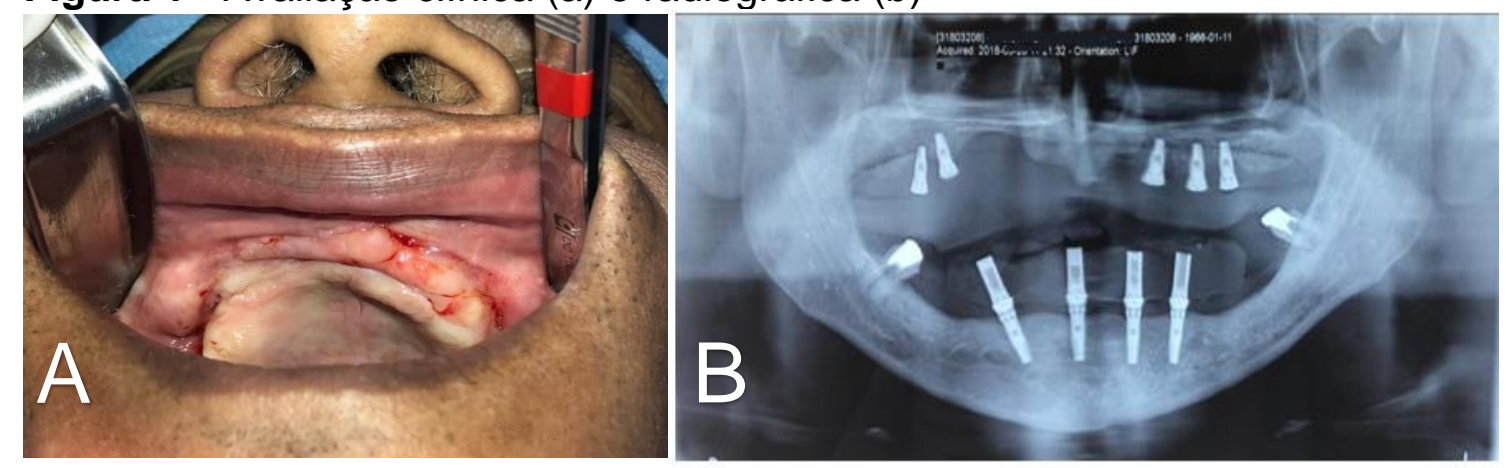

Fonte: os autores.

Foram realizados exames pré-operatórios de rotina, exames laboratoriais como hemograma completo, coagulograma, glicemia, uréia, creatinina, radiografia de tórax, pressão arterial (PA),eletrocardiogramaeencaminhados para a consulta pré anestésica no Hospital Manoel Victorino, onde ocorreu a cirurgia com equipe multidisciplinar, incluindo cirurgião bucomaxilofacial e ortopedista. A cirurgia foi realizada sob anestesia geral com intubação nasotraqueal,inicialmente o preparo do acesso foi feito em área doadora com a remoção do enxerto da crista ilíaca, que foi realizada pela equipe de ortopedia do hospital. 
A cirurgia foi realizada por acesso na crista do rebordo alveolar de espessura total tipo mucoperiosteal, com duas incisões relaxantes vestibulares em região da tuberosidade da maxila. Após exposição do defeito foram removidos os implantes antigos com osteointegração parcial e desbridamento de toda a área a ser enxertada.

O bloco ósseo removido da crista ilíaca foi cortado em vários fragmentos cúbicos de $2 \times 2 \mathrm{~cm}$ em média (Figura 2a), sem preparo da crista ilíaca e compressão, apenas a raspagem do periósteo, e fixados com parafusos bicorticais do sistema 2.0 (Figura 2b). Foram realizadas incisões no periosteo com objetivo de aumentar a mobilidade do retalho para realizar 0 reposicionamento do mesmo sem tensão e síntese dos tecidos, com sutura tipo ponto simples utilizando fios monofilamentado n. 4-0 (nylon) (Figura 2c).

Figura 2 - Blocos ósseos removidos da crista ilíaca em fragmentos cúbicos de $2 \times 2 \mathrm{~cm}$ (a); Bloco ósseo fixados com parafusos bicorticais (b); Suturas de ponto simples com fio 4-0 Nylon (c)

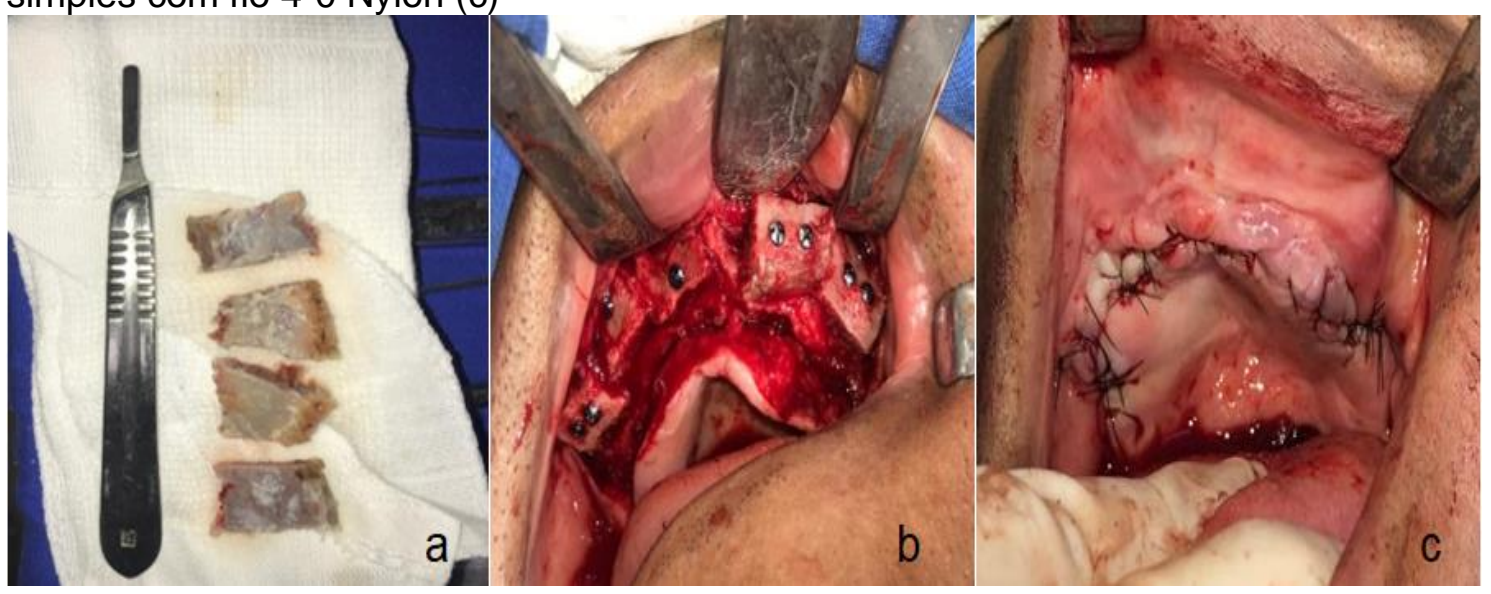

Fonte: os autores.

O paciente evoluiu bem, sem queixas, aceitando a dieta, deambulando com ajuda de andador nos primeiros 10 dias, sem sinais de infecção, recebendo alta no segundo dia pós operatório, após remoção do dreno em área doadora. $\mathrm{O}$ mesmo foi orientado a permanecer sem prótese e manter dieta líquida pastosa por 90 dias até a data para instalação dos implantes. Os medicamentos receitados no pós-operatório foram: amoxicilina ${ }^{\circledR}$ de $875 \mathrm{mg} \mathrm{em} 12$ em 12 horas durante 14 dias devido a comunicação com seio maxilar; nimesulida ${ }^{\circledR} 100 \mathrm{mg}$ em 12 e 12 horas durante 5 dias; dipirona $\AA 1 \mathrm{~g}$ em 6 e 6 horas durante 2 dias e realizar a higienização com Periogard®, banhar a região com $15 \mathrm{ml}$, por 1 minuto, 2 vezes ao dia, 30 minutos após a escovação, durante 7 dias.

As suturas foram removidas após 15 dias em ambos os leitos, onde se evidenciou uma boa cicatrização sem exposição do material de enxertia. Após três meses foi confeccionado o guia cirúrgico e realizada a tomografia computadorizada de feixe cônico para controle pós-operatório e planejamento para instalação dos implantes (Figura 3a, b, c). Na instalação dos implantes, a cirurgia foi realizada sob anestesia local com acesso similar para remoção dos parafusos de fixação do enxerto e instalação de 8 implantes na maxila. 
Figura 3 - Radiogafia panorâmica para o planejamento da instalação dos implantes (a); Incisão relaxante na vestibular em região da tuberosidade da maxila (b); Instalação dos implantes com guia cirúrgicos (c)

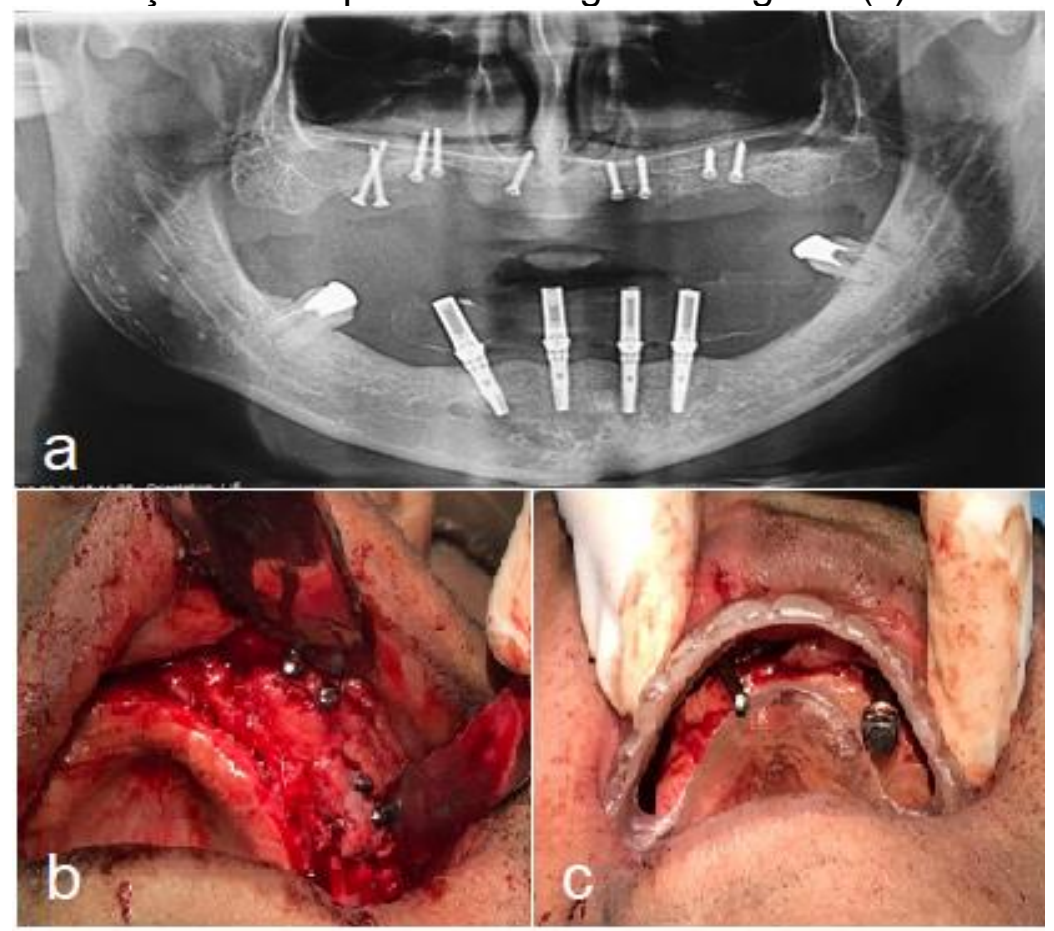

Fonte: os autores.

O paciente evoluiu bem, sem queixasesem sinais de infecção. Após seis meses foi realizado o segundo estágio para colocação de cicatrizadores, onde foi observada a perda de um implante. Atualmente o mesmo encontra-se na fase de acompanhamento e já foi encaminhado para a confecção da prótese definitiva.

\section{DISCUSSÃO}

Mesmo sendo um tratamento extenso, a reconstrução de maxila atrófica através do enxerto ósseo tem um alto índice de sucesso, corrigindo a estética e principalmente a sua função (ROMERO et al., 2009).

Na literatura GAZDAG et al., (1995) apresenta alguns tipos de enxertos ósseos para a reconstrução das atrofias maxilares como: homólogo, heterógeno, aloplástico e o autógeno que é o de primeira escolha, pois é considerado padrão ouro pelos cirurgiões dentistas devido a sua biocompatibilidade, osteoindução e osteocondutividade, proporcionando uma integração óssea maior, sendo mais previsível, rápida e com uma nova formação óssea densa e madura entre 6 e 12 meses após a sua enxertia.

O enxerto autógeno, sendo área doadora intrabucal, devemos aguardar a formação óssea entre 6 a 12 meses, no enxerto extrabucal, principalmente da região da crista ilíaca, por apresentar alto indice de reabsorção, é indicado instalar os implantes com 3 meses após a sua enxertia (SOUZA et al., 2019). 
O enxerto autógeno é dividido em intrabucal e extrabucal, a área escolhida para ser doadora depende da quantidade de osso. Para pequenos e médios defeitos é indicado intrabucal, em grandes reconstruções que são necessárias uma boa quantidade de tecido ósseo, a indicação para a remoção dos enxertos são as áreas extrabucais (KLASSMANN et al., 2006).

No caso apresentado foi indicado a utilização de enxerto autógeno extra bucal, devido a necessidade de grande volume de material e pelo fato deo paciente já haver sido submetido a outros métodos de reconstrução onde houve insucesso.Por se tratar de uma reconstrução total da arcada superior e com uma atrofia severa, a área escolhida para ser doadora foi a crista ilíaca, pois ela fornece grande quantidade do tecido ósseo e auxilia na reconstrução maxilar tanto na altura e espessura,como na elevação do assoalho do seio maxilar. Realizada a instalação dos implantes com 90 dias devido ao alto indice de reabsorção óssea da região da crista ilíaca.

Durante a remoção do tecido doador da crista ilíaca, pode ocorrer complicação pós-operatória que geralmente é relacionada a grande quantidade de osso retirado, podendo ocorrer hemorragias internas, edema e dor. A imprudência do cirurgião pode afetar alguma estrutura anatômica do paciente, como o nervo lateral femural cutâneo causando sua ruptura, o que faz com que o paciente tenha parestesia parcial ou definitiva da coxa na sua porção lateral,dificultando sua locomoção, porém neste caso, não houve nenhuma complicação pós-operatória (SJOSTROM et al., 2007; SBORDONE et al., 2009; ROCHA et al., 2015).

Observaram como desvantagem, a morbidade pós-operatória devido as limitações de locomoção temporária, o que não foi observado no caso clínico em questão. O paciente, no primeiro dia pós-operatório, já deambulava com ajuda de andador e após dez dias não relatava dificuldade ou sintomatologia ao caminhar (SJOSTROM et al., 2007; SBORDONE et al., 2009; ROCHA et al., 2015).

Ocorre a realização de um estudo pré-operatório clínico e radiográfico que determinará o tamanho do defeito ósseo e a quantidade necessária para a sua reconstrução. Salvato e Agliardi (2007) e Ella et al., (2008), observaram que para o estudo pré-operatório, o uso da tomografia computadorizada é importante, pois é realizado um estudo em 3D que permite um planejamento apurado, avaliando quantidade e qualidade do osso.

Segundo Mannai (2006) o uso de barreiras membranosas limita a migração do tecido mole para o local do enxerto,fazendo com que ocorra melhoria na cicatrização óssea limitandoa reabsorção óssea. Neste caso não foram utilizadas membranas, pois não havia a membrana disponível no local da cirurgia. A reabsorção óssea após 3 meses foi considerada compatível com o tipo de biomaterial, não prejudicando a instalação dos implantes.

Segundo Prejrone et al. (2002) é importante que o paciente seja orientado a não utilizar nenhum tipo de prótese para que não ocorra compressão do enxerto, assim evitando a sua reabsorção. Neste caso clínico, além do paciente não utilizar a prótese, ele foi orientado a manter uma alimentação pastosa por 90 dias. Logo após foram colocados os implantes e em seguida confeccionada nova prótese total com alívio na região da crista do rebordo. 


\section{CONSIDERAÇÕES FINAIS}

Apesar da evolução e do fácil acesso aos biomateriais, o enxerto de crista ilíaca ainda é uma excelente opção nos casos de defeitos ósseos severos, com boa previsibilidade, baixo índices de complicações e rápido período de incorporação.

\section{REFERÊNCIAS}

ELLA, B. et al. Septa within the sinus: effect on elevation of the sinus floor. British Journal of Oral and Maxillofacial Surgery, v. 46, n. 6, p. 464467, 2008.

FLORIAN, F. et al. Complicações Associadas aos Enxertos Ósseos Aposicionais com Osso Autógeno. Revista Brasileira de Cirurgia buco-maxilo-facial, v. 10, n. 2, p. 15-22, 2010.

GAZDAG, A. et al. Alternativesto Autogenous Bone Graft: Efficacy and Indications. Journal of the American Academy of Orthopaedic Surgeons, v. 3, n. 1, p. 1-8, 1995.

KLASSMANN, F. A. et al. Enxertos ósseos autógenos de áreas doadoras intrabucais e procedimentos clínicos integrados possibilitando reabilitação estética e funcional. Revista Gaúcha de Odontologia, v. 54, n. 4, p. 388-392, 2006.

LEVIN, L.; NITZAN, D. I.; ARAD, D. S. Success of Dental Implants Placedin Intraoral Block Bone Grafts. Journal of Periodontology, v. 78, n. 1, p. 18-21, 2007.

MANNAI, C. Early Implant Loading in Severely Resorbed Maxilla Using Xenograft, Autograft, and Platelet-Rich Plasma in 97 Patients. Journal of Oral and Maxillofacial Surgery, v. 64, n. 1, p. 1420-1426, 2006.

PALECKIS, L. G. P. et al. Enxerto ósseo autógeno: por que e como utilizá-lo. Revista Implant News, v. 2, n. 4, p. 369-374, 2005.

PEJRONE, G. et al. Sinus floor augmentation with autogenous iliac bone block grafts: a histological and histomorphometrical report on the two-step surgical technique. International Journal of Oral and Maxillofacial Surgery, v. 31, n. 4, p. 383-388, 2002.

ROCHA, J. F. et al. Enxerto ósseo mandibular, complicações associadas às áreas doadoras e receptoras, e sobrevivência de implantes dentários: um estudo retrospectivo. Revista Odontologia UNESP, v. 44, n. 6, p. 340-344, 2015.

ROMERO, R. L. et al. Implantes zigomáticos $x$ reconstrução de maxila com enxerto de iliáco - relato de caso clínico. Periodontia, v. 19, n. 4, p. 82-88, 2009. 
SALVATO, G.; AGLIARDI, E. Calvarial Bone Grafts in Severe Maxillary Atrophy: Preprosthetic Surgery with Sedation. Implant Dentistry, v. 16, n. 4, p. 356-359, 2007.

SBORDONE, L. et al. Volume changes of autogenous bone grafts after alveolar ridge augmentation of atrophic maxillae and mandibles. International Journal of Oral and Maxillofacial Surgery, v. 38, n. 10, p. 1059-1065, 2009.

SILVA, G. H. S. et al. Reconstrução de maxila atrófica com enxerto da crista ilíaca: revista da literatura e apresentação de caso clínico cirúrgico. Revista Eletrônica de Odontologia da Academia Tiradentes de Odontologia, v. 7, n. 10, p. 871-886, 2007.

SJÖSTRÖM, M. et al. Reconstruction of the Atrophic Edentulous Maxilla with Free lliac Crest Grafts and Implants: A 3-Year Report of a Prospective Clinical Study. Clinical Implant Dentistry and Related Research, v. 9, n. 1, p. 46-59, 2007.

SOUZA, C. S. et al. Analysis of the Survival of Dental Implants Installed in Reconstructed Maxilla with Autogenous lliac Crest Graft: 7- to 9-Year Follow-Up. Journal Oral Implanto, v. 45, n. 6, p. 427-436, 2019.

WORTHINGTON, P.; LANG, B.; RUBENSTEIN, J. Osseointegração em Odontologia: Uma Visão Geral., 2. ed. 174p. Editora Quintessence. 2005. 